Proceedings of the Edinburgh Mathematical Society (2008) 51, 1-9 (C)

DOI:10.1017/S001309150500060X Printed in the United Kingdom

\title{
d-FRATTINI CHIEF FACTORS
}

\author{
H. BECHTELL \\ Mathematics Department, University of New Hampshire, \\ Durham, NH 03824, USA (bechtell@cisunix.unh.edu)
}

(Received 26 April 2005)

\begin{abstract}
The concepts of d- and nd-Frattini chief factors of a finite group are introduced. Their ingrainment into that of the extended Frattini dual subgroup becomes the natural dual to Frattini and supplemented chief factors. Not only does a dual of the strengthened form of the Jordan-Hölder theorem arise, but also the $p$-nilpotent radical becomes the intersection of the centralizers of the nd-Frattini chief factors. As a result, a class $\mathfrak{F}$ of groups is a full integrated local formation $\operatorname{LF}(f)$ if and only if each nd-Frattini chief factor in $G \in \mathfrak{F}$ is $f$-central.
\end{abstract}

Keywords: extended Frattini dual; d-Frattini chief factors; saturated formations

2000 Mathematics subject classification: Primary 20D25

Secondary 20D10

\section{Introduction}

This article establishes the role of the minimal subgroups in the structure of a finite group in a manner 'dual' to that of the maximal subgroups.

The focus is primarily on the extended Frattini dual $\Psi_{\mathrm{e}}(G)$ and its structure as a product of the normal closures of minimal subgroups and cyclic subgroups of order 4 . These normal closures suggest the examination of a chief factor $H / K$ in a group as being either a d-Frattini chief factor, $\Psi_{\mathrm{e}}(H)=\Psi_{\mathrm{e}}(K)$, or an nd-Frattini chief factor, $H=K \omega_{\mathrm{e}}(x)$, for the normal closure $\omega_{\mathrm{e}}(x)$ of an element $x$ of either odd prime order or order that divides 4 . These 'duals' to the Frattini and supplemented chief factors, respectively, yield an analogue of the strengthened form of the Jordan-Hölder theorem. One result is that the centralizer of those nd-Frattini chief factors of the group which are contained in $\Psi_{\mathrm{e}}(G)$ and for which $|x|$ is either an odd prime or $|x|$ divides 4 is the $p$-nilpotent radical. A local definition on the automorphism groups of these nd-Frattini chief factors within $\Psi_{\mathrm{e}}(G)$ provides an alternative definition for a local formation. This extends the results of Laue $[\mathbf{6}]$, which were the motivation for the approach introduced here.

The notation and terminology in this paper will, for the most part, be that found in the standard references $[\mathbf{2}]$ and [4] with the following exception. For brevity and clarity, the use of terms such as $G$-normal subgroup, $G$-chief factor and $G$-chief series highlights 
$G$ as the set of operators and avoids potential conflict related with subnormality. For a subgroup $S$ in a group $G$, the normal closure $\left\langle S^{g} \mid g \in G\right\rangle$ is denoted simply by $S^{G}$.

\section{2. $\omega_{\mathrm{e}}$-subgroups and chief factors}

The Frattini dual $\Psi(G)$ of a group $G$ is the subgroup generated by the minimal subgroups in $G$, i.e. $\Psi(G)=\langle S|S \subseteq G| S \mid, \in \pi(G)\rangle, \pi(G)$ the set of all primes that divide $|G|$ and the extended Frattini dual $\Psi_{\mathrm{e}}(G)=\langle S \subseteq G,|S| \in \pi(G)$ or $S$ is cyclic of order 4$\rangle$. The focus of this article is on formalizing the role of the normal closures $S^{G}$ of these subgroups within the group structure. An unexpected consequence is an apparent dualization of concepts identified with the Frattini subgroup.

Since $\Psi(G) \subseteq \Psi_{\mathrm{e}}(G)$, the notation must reflect which dual is under consideration.

For a subgroup $T$ in a group $G$ and an element $x \in T$ of prime order $p$, denote the $T$ normal hull $\langle x\rangle^{T}$ by $\omega_{T}\left(x \mid x^{p}=1\right)$ or simply $\omega_{T}(x)$, with the understanding that $x$ has prime order. Hence, $\omega_{T}(x) \subseteq \Psi(T)$. If attention is directed to $\Psi_{\mathrm{e}}(T)$, then $\omega_{T \mathrm{e}}\left(x \mid x^{n}=1\right)$ denotes $\langle x\rangle^{T}$ and $n$ may be either an odd prime or a divisor of 4 . The abbreviated form will be $\omega_{T \mathrm{e}}(x)$. We refer to these $T$-normal closures as either $\omega_{T^{-}}$or $\omega_{T \mathrm{e}}$-subgroups for $x \in T$. So $\omega_{T}(x) \subseteq \Psi(T)$ implies that $\omega_{T}(x)=\omega_{T}\left(x \mid x^{n}=1\right)$ for $n \in \mathbb{P}$, where $\mathbb{P}$ is the set of primes, and $\omega_{T \mathrm{e}}(x) \subseteq \Psi_{\mathrm{e}}(T)$ implies that $\omega_{T \mathrm{e}}(x)=\omega_{T \mathrm{e}}\left(x \mid x^{n}=1\right)$ for $n \in \mathbb{Q}=\mathbb{P} \cup\{4\}$. Whenever $T=G$, our primary interest, we use $\omega(x)$ and $\omega_{\mathrm{e}}(x)$ and refer to them as $\omega$-subgroups and $\omega_{\mathrm{e}}$-subgroups of $G$, respectively.

In this notation, $\Psi(T)=\prod\left\{\omega_{T}(x) \mid x^{n}=1, n \in \mathbb{P}\right\}$, the product over all minimal subgroups in $T$, and $\Psi_{\mathrm{e}}(T)=\prod\left\{\omega_{T \mathrm{e}}(x) \mid x^{n}=1, n \in \mathbb{Q}\right\}$, the product over all minimal subgroups in $T$ and the cyclic subgroups in $T$ of order 4 . In abbreviated form,

$$
\Psi(T)=\prod_{\mathbb{P}}\left\{\omega_{T}(x)\right\} \quad \text { and } \quad \Psi_{\mathrm{e}}(T)=\prod_{\mathbb{Q}}\left\{\omega_{T \mathrm{e}}(x)\right\}
$$

Since $x \in \omega_{T}(x)$, we find that $\omega_{N}(x) \subseteq \omega_{T}(x)$ is subnormal in $\omega_{T}(x)$, for $N=\omega_{T}(x)$. The process is iterative. Setting $\omega_{T}(x)=\omega_{T}^{1}(x)$, define $\omega_{T}^{j}(x)=\omega_{N}(x)$ for $N=\omega_{T}^{j-1}(x)$. There exists a subnormal chain

$$
\omega_{T}(x)=\omega_{T}^{1}(x) \supseteq \cdots \supseteq \omega_{T}^{j}(x) \supseteq \cdots \supset\{1\}
$$

referred to as an $\omega$-chain in $T$. Since $|G|$ is finite and $\omega_{T}^{j}(x) \neq\{1\}$ for any integer $j$, there exists a minimal integer $k$ such that $\omega_{T}^{j}(x)=\omega_{T}^{k}(x)$ for all $j \geqslant k$.

A subgroup $\omega_{T}(x)$ is said to be $d$-primitive with respect to $T$ if $\omega_{T}(x)=\omega_{T}^{2}(x)$. In the above subnormal chain, $\omega_{T}^{k}(x)$ is d-primitive.

These subgroups are introduced at this point, since they highlight the fact that the duality between the core of a maximal subgroup and the normal closure of a minimal subgroup does not imply duality with respect to each property associated with the core.

Consequently, for a subgroup $T$ in $G, \omega_{T}(x)=\left\langle\omega_{T}^{k}(x)\right\rangle^{T}$ for the terminal member $\omega_{T}^{k}$ in an $\omega$-chain. Hence, $\omega_{T}(x)$ is the normal closure in $T$ of a d-primitive group.

A similar development evolves with respect to $\omega_{T \mathrm{e}}(x)$ with an appropriate adjustment in terminology and notation. 
For a subgroup $N \unlhd G, \Psi_{N \mathrm{e}}(N)$ is the product of the $\omega_{N \mathrm{e}}(x)$ over all minimal subgroups and cyclic subgroups of order 4 in $N$, which implies that $\Psi_{N \mathrm{e}}(N)$ is the product of the $\omega_{\mathrm{e}}(x)$ over the same set of elements, since $\Psi_{N \mathrm{e}}(N) \unlhd G$. Since our focus is on the $\omega_{\mathrm{e}}$-subgroup $\omega_{\mathrm{e}}(x), \Psi_{\mathrm{e}}(N)$ rather than $\Psi_{N \mathrm{e}}(N)$ will be used.

In general, for $N \unlhd G$, we have $N \cap \Psi_{\mathrm{e}}(G) \neq \Psi_{\mathrm{e}}(N)$. For example, consider

$$
G=\left[\left\langle x \mid x^{9}=1\right\rangle\right]\left\langle y \mid y^{2}=1\right\rangle
$$

and $y^{-1} x y=x^{-1}$. Then $G=\omega_{\mathrm{e}}(y)=\Psi_{\mathrm{e}}(G)$ and $\langle x\rangle \cap G=\langle x\rangle \neq \Psi_{\mathrm{e}}(\langle x\rangle)=\left\langle x^{3}\right\rangle$. On the other hand, for $N \unlhd G, \Psi_{\mathrm{e}}\left(N \cap \Psi_{\mathrm{e}}(G)\right)=\Psi_{\mathrm{e}}(N)$.

The dual of a $G$-chief factor $H / K \subseteq \Phi(G / K)$, the intersection of all maximal subgroups in $G$ that contain $H$, is that of a $G$-chief factor $S / T$ such that $\Psi_{\mathrm{e}}(S) \subseteq T$. The fact that $H / K$ cannot be supplemented in $G / K$ by a proper subgroup dualizes to the fact that $S$ does not contain a non-trivial subgroup $U$ such that $T \cap U=\{1\}$. For a subgroup $N \unlhd G$, the dual to $N \Phi(G) / N \subseteq \Phi(G / N)$ becomes $\Psi_{\mathrm{e}}(G) \cap N \supseteq \Psi_{\mathrm{e}}(N)$.

Attention will be restricted to the extended Frattini dual $\Psi_{\mathrm{e}}(G)$.

Definition 2.1. A $G$-chief factor $S / T$ in a group $G$ is a $d$-Frattini chief factor if $\Psi_{\mathrm{e}}(S) \subseteq T$ and is an nd-Frattini chief factor if $S=T \Psi_{\mathrm{e}}(S)$.

Consequently, a d-Frattini chief factor $S / T$ has $\Psi_{\mathrm{e}}(S)=\Psi_{\mathrm{e}}(T)$. The concept is well defined and the two sets are complements in the collection of all $G$-chief factors in a group $G$. No minimal $G$-normal subgroup can be a d-Frattini chief factor nor can $T$ be complemented in $S$ for a d-Frattini chief factor $S / T$. Each $G$-chief factor $S / T$ such that $\Psi_{\mathrm{e}}(G) \subseteq T$ is a d-Frattini chief factor and $\Psi_{\mathrm{e}}(S)=\Psi_{\mathrm{e}}(T)=\Psi_{\mathrm{e}}(G)$. On the other hand, each $G$-chief factor $\Psi_{\mathrm{e}}(G) / K$ is an nd-Frattini chief factor.

Even though $\Psi(S) \subseteq \Psi_{\mathrm{e}}(S)$, each $G$-chief factor in $G=\Psi_{\mathrm{e}}(G) \cong Q_{8}$ is nd-Frattini relative to $\Psi_{\mathrm{e}}(G)$, but this is not the case with respect to $\Psi(G)$.

The property of a $G$-chief factor being Frattini is preserved under epimorphism but not in general with respect to containment in a $G$-normal subgroup. Just the opposite is valid for d-Frattini chief factors. The non-abelian $p$-group of order $p^{3}$, exponent $p^{2}$, for $p>2$, contains examples showing that the $G$-isomorphism alone does not preserve $G$-chief factors that are either d-Frattini or nd-Frattini.

Proposition 2.2. For a subgroup $N$ normal in a group $G$,

(a) each $G$-chief factor in $N / \Psi_{\mathrm{e}}(N)$ is d-Frattini and

(b) each $G$-chief factor $\Psi_{\mathrm{e}}(N) / T$ is nd-Frattini.

From Definition 2.1, a $G$-chief factor $S / T$ is nd-Frattini if and only if

$$
S=T \omega_{\mathrm{e}}\left(x \mid x^{n}=1\right)
$$

for $n \in \mathbb{Q}$ and $\langle x\rangle \subseteq S \backslash T$. In this case $\omega_{\mathrm{e}}(x)$ is said to be a cap for $S / T$, or $S / T$ is capped by $\omega_{\mathrm{e}}(x)$. 
Proposition 2.3. Consider non-trivial normal subgroups $N, M, K$ and $R=N \cap M$ in a group $G$ such that $K / N$ and $K / M$ are $G$-chief factors and $N \neq M$. Then $K / N \cong_{G} M / R$ and $K / M \cong_{G} N / R$.

(a) $K / N$ being $d$-Frattini and $K / M$ being nd-Frattini imply that $M / R$ is $d$-Frattini and $N / R$ is nd-Frattini and the converse.

(b) $K / N$ and $K / M$ are $d$-Frattini if and only if $N / R$ and $M / R$ are $d$-Frattini.

(c) $N / R$ and $M / R$ are nd-Frattini if and only if $K / N$ and $K / M$ are nd-Frattini.

Proof. For (a), if $M / R$ is nd-Frattini, there exists $\langle x\rangle \subseteq M \backslash R$ such that $M=R \omega_{\mathrm{e}}(x)$. But $\omega_{\mathrm{e}}(x) \subseteq \Psi_{\mathrm{e}}(K)=\Psi_{\mathrm{e}}(N)$ implies that $\langle x\rangle \subseteq R$, which is a contradiction. So $M / R$ is d-Frattini. If $K / M$ is nd-Frattini, then $K=M \omega_{\mathrm{e}}(x)$ for $\langle x\rangle \subseteq K \backslash M$. However, $\langle x\rangle \subseteq \Psi_{\mathrm{e}}(K)=\Psi_{\mathrm{e}}(N)$ implies $\langle x\rangle \subseteq N \backslash R$. So $N=R \omega_{\mathrm{e}}(x)$, i.e. $N / R$ is nd-Frattini. Due to the symmetry of the argument, the conditions are necessary and sufficient. Then (b) and (c) become corollaries of (a).

This leads to a dual of the strengthened form of the Jordan-Hölder theorem.

Proposition 2.4. For two $G$-chief series $\mathcal{S}_{1}$ and $\mathcal{S}_{2}$ in a subgroup $T$ that is normal in a group $G$, there exists a bijection between the $G$-chief factors of $\mathcal{S}_{1}$ and those of $\mathcal{S}_{2}$ such that the corresponding factors are $G$-isomorphic and the d-Frattini chief factors of $\mathcal{S}_{1}$ correspond to the d-Frattini chief factors of $\mathcal{S}_{2}$.

Proof. $\mathcal{S}_{1}$ and $\mathcal{S}_{2}$ have the same length $n$ by the Jordan-Hölder theorem on $\Omega$-groups (see, for example, $[\mathbf{2}, \S$ A.3.2]) for $\Omega=\operatorname{Inn}(G)$. Induction will be used for $n>1$ since the result is trivially valid for $n=1$. If $T$ contains precisely one subgroup $M$ maximal with respect to being $G$-normal and contained in $T$, the conclusion follows by induction on $M$.

Suppose that $T$ contains two $G$-normal subgroups $S_{1}$ and $T_{1}$ properly contained in $T$ and maximal with respect to being $G$-normal. Consider two $G$-chief series for $T$,

$$
\mathcal{S}_{1}: T=S_{0} \supset S_{1} \supset \cdots \supset S_{r}=\{1\} \quad \text { and } \quad \mathcal{S}_{2}: T=T_{0} \supset T_{1} \supset \cdots \supset T_{s}=\{1\} .
$$

By the Jordan-Hölder theorem, $r=s$. If $R=S_{1} \cap T_{1}=\{1\}$, the result is valid (by Proposition 2.3). Otherwise, consider the $G$-chief series $\mathcal{U}: T \supset S_{1} \supset R=R_{2} \supset \cdots \supset$ $R_{s-2}=\{1\}$ and also $\mathcal{V}: T=V_{0} \supset V_{1}=T_{1} \supset R=R_{2} \supset \cdots \supset R_{s-2}=\{1\}$. By induction, the theorem is valid for the two series

$$
S_{1} \supset \cdots \supset S_{s}=\{1\} \quad \text { and } \quad S_{1} \supset R \supset \cdots \supset R_{s-2}=\{1\}
$$

as well as for

$$
T_{1} \supset \cdots \supset T_{s}=\{1\} \quad \text { and } \quad T_{1} \supset R \supset \cdots \supset R_{s-2}=\{1\} .
$$

By the application of Proposition 2.3 to $T, S_{1}, T_{1}$ and $R$, we have $T / T_{1} \cong_{G} S_{1} / R \cong_{G} F_{1}$, where $F_{1}$ is a factor in $\mathcal{S}_{1}$ below $S_{1}$, and $T / S_{1} \cong_{G} T_{1} / R \cong_{G} F_{2}$, where $F_{2}$ is a factor in $\mathcal{S}_{2}$ below $T_{1}$. The conclusion follows. 
On the domain of finite groups, let $\Gamma$ denote the functor (class function) on $\Sigma(G)$, the lattice of $G$-normal subgroups contained in the group $G$ such that $N \in \Gamma(G)$ if and only if either $N=\{1\}$ or each $G$-chief factor $H / K \in N$ is nd-Frattini. We refer to $\Gamma(G)$ as a $\Gamma$-set for $G$.

Consider $K=N M$ for the non-trivial subgroups $N, M \in \Gamma(G)$ and the $G$-chief factor $A / N \cap M \subseteq M / N \cap M \cong_{G} K / N$. By hypothesis, $A=(N \cap M) \omega_{\mathrm{e}}(x)$. Then $N A / N \cong_{G}$ $N \omega_{\mathrm{e}}(x) / N$ is nd-Frattini, for otherwise $\langle x\rangle \subseteq N$ leads to a contradiction. Inductively there exist an nd-Frattini chief factor $S / T \in M$ such that $S=T \omega_{\mathrm{e}}(x), N \cap M \subseteq$ $T \subset S \subseteq M$, and a $G$-chief series from $N$ to $N T$ consisting of nd-Frattini chief factors. Moreover, $N S / N T=S(N T) / N T \cong_{G} S / S \cap N T=S / T(S \cap N)=S / T$. If $x \in N$, then $x \in N \cap M \subseteq T$, which is a contradiction. Hence, $\omega_{\mathrm{e}}(x)$ caps $N S / N T$, that is, $N S / N T$ is nd-Frattini. Consequently, there exists a $G$-chief series through $N$ to $K$ in which each $G$-chief factor is nd-Frattini. By Proposition 2.4, each $G$-chief factor in $K$ is nd-Frattini; so $K \in \Gamma(G)$. From the definition, each $G$-normal subgroup $L \subseteq N \in \Gamma(G)$ is in $\Gamma(G)$.

Each $\theta \in \operatorname{Aut}(G)$ preserves $G$-chief factors. If $H / K$ is a $G$-chief factor such that $H=K \omega_{\mathrm{e}}(x)$, then $x^{\theta} \in K^{\theta}$ implies that $x \in K$, which is a contradiction. So $\Gamma(G)$ is a well-defined characteristic subset of $\Sigma(G)$.

The following proposition gives a summary.

Proposition 2.5. A $\Gamma$-set of a group $G$ satisfies the following properties:

(a) $\Gamma(G)$ is a well-defined characteristic subset of $\Sigma(G)$;

(b) if $T$ is a $G$-normal subgroup contained in $S \in \Gamma(G)$, then $T \in \Gamma(G)$;

(c) for subgroups $N$, we have $M \in \Gamma(G)$ and $N M \in \Gamma(G)$.

Note that (b) and (c) can be combined as follows. The subgroups $N, M \in \Gamma(G)$ if and only if $N M \in \Gamma(G)$.

Since $\Gamma(G) \neq \emptyset$ for any group $G$, and $G$ is of finite order, the next result is valid by Definition 2.1 and through induction.

Proposition 2.6. With respect to a $\Gamma$-set $\Gamma(G)$, there exists a unique characteristic subgroup $G_{\Gamma}$, the $\Gamma$-radical, such that $N \in \Gamma(G)$ if and only if $N \subseteq G_{\Gamma}$.

Since subnormality is not involved, a $\Gamma$-set is not a Fitting class.

This concept is analogous to that of the $\boldsymbol{n} \boldsymbol{C}$-groups $\mathfrak{F}$ introduced by Christensen $[\mathbf{1}]$ groups that split over each normal subgroup - and was proven to be a formation in [5].

The structure of the finite $\boldsymbol{n} \boldsymbol{C}$-groups has not been characterized in general. Note that an $\boldsymbol{n} \boldsymbol{C}$-group $G$ is equal to $G_{\Gamma}$ since, for each $G$-chief factor $H / K, G=[K] L$ implies that $H=[K](H \cap L)=K \omega_{\mathrm{e}}(x)$ for an element $x \in H \cap L$ of either prime order or order dividing 4 . As a cyclic group of order 4 indicates, the converse is not valid. However, the soluble multiprimitive groups $\mathfrak{P}^{Q}$ of Hawkes $[\mathbf{3}, \S 2]$ is an exception. A soluble group $G \in \mathfrak{P}^{Q}$ if and only if $G$ has a unique $G$-chief series and each $G$-chief factor is complemented. As a result, each $G$-normal subgroup $H$ is contained in this series. For a $G$-chief factor $H / K$, we have $H=K \omega_{\mathrm{e}}(x)$. But $\omega_{\mathrm{e}}(x)$ is in the series and not in $K$. Therefore, $H=\omega_{\mathrm{e}}(x)$. Conversely, suppose that $G$ has a unique $G$-chief series such that 
each member $N$ is equal to $\omega_{\mathrm{e}}\left(x \mid x^{p}=1\right)$ for some prime $p$. Since the result is valid for all groups of prime order and the hypothesis is valid for each non-trivial epimorphic image whenever the series is of length greater than one, we use induction on the length of the series. Consider the unique minimal normal subgroup $M$ in $G$. By $[\mathbf{3},(2.2)], G / M \in \mathfrak{P}^{Q}$ implies $G \in \mathfrak{P}^{Q}$. This verifies the next proposition.

Proposition 2.7. A necessary and sufficient condition for a soluble group $G$ to be multiprimitive is that $G$ contains a unique $G$-chief series $\mathcal{S}$ in which each $H \in \mathcal{S}$ is an $\omega_{\mathrm{e}}$-subgroup of the form $\omega_{\mathrm{e}}\left(x \mid x^{p}=1\right)$ for a prime $p$ that divides the order of the chief factor $H / K$.

A dual to a $\Gamma$-set is a collection $\mathfrak{f}$, the $\mathfrak{f}$-set, that consists of factor groups of a group $G$ in which either each factor group $G / N$ is $G / G$ or each $G$-chief factor in $G / N$ is d-Frattini, i.e. $\Psi_{\mathrm{e}}(G)=\Psi_{\mathrm{e}}(N)$. Therefore, for $M / N \unlhd G / N$, we have $G / N \in \mathfrak{f}(G)$, and $\Psi_{\mathrm{e}}(N) \subseteq \Psi_{\mathrm{e}}(M) \subseteq \Psi_{\mathrm{e}}(G)$ implies that $\Psi_{\mathrm{e}}(M)=\Psi_{\mathrm{e}}(G)$. Furthermore, $G / N, G / M \in \mathfrak{f}(G)$ implies that $\Psi_{\mathrm{e}}(G)=\Psi_{\mathrm{e}}(N)=\Psi_{\mathrm{e}}(M) \subseteq N \cap M$. Hence, $\Psi_{\mathrm{e}}(G)=\Psi_{\mathrm{e}}(N \cap M)$. Therefore, each $G$-chief factor in $G / N \cap M$ is d-Frattini, i.e. $G / N \cap M \in \mathfrak{f}(G)$. Since $G$ is finite, there exits by induction a unique $G$-normal subgroup $G^{\mathfrak{f}}$ such that $G / N \in \mathfrak{f}(G)$ if and only if $G^{\mathfrak{f}} \subseteq N$. Since d-Frattini chief factors are preserved with respect to automorphisms, $G^{\mathfrak{f}}$ is characteristic in $G$. In particular, $G^{\mathfrak{f}}=\Psi_{\mathrm{e}}(G)$.

Proposition 2.8. An $\mathfrak{f}$-set for a group $G$ satisfies the following properties:

(a) $\mathfrak{f}(G)$ is a well-defined characteristic subset of factor groups in $G$;

(b) if $G / T$ is a factor group and $M / T \unlhd G / T \in \mathfrak{f}(G)$, then $G / M \in \mathfrak{f}(G)$;

(c) for factor groups $G / N, G / M \in \mathfrak{f}(G)$, we have $G / N \cap M \in \mathfrak{f}(G)$;

(d) the factor group $G / N \in \mathfrak{f}(G)$ if and only if $\Psi_{\mathrm{e}}(G) \subseteq N$.

Note that an $\mathfrak{f}$-set is not a formation since $G / N, G / M \in \mathfrak{f}(G)$ and $N \cap M=\{1\}$ can occur only if $|G|=1$.

This is dual to the collection of normal subgroups in a group $G$, having each $G$-chief factor Frattini, in which $\Phi(G)$ is the 'radical'.

Consider a $G$-chief series $\{1\}=H_{t} \subset \cdots \subset H_{0}=N$ within a $G$-normal subgroup $N \neq\{1\}$ in a group $G$. Since $H_{t-1}$ is minimal normal in $G, H_{t-1} \subseteq \Psi_{\mathrm{e}}(N)$. If $H_{t-2} / H_{t-1}$ is d-Frattini, then $\Psi_{\mathrm{e}}\left(H_{t-2}\right)=H_{t-1}$. If $H_{t-2} / H_{t-1}$ is nd-Frattini, there exists at least one $\omega_{\mathrm{e}}(x) \subseteq H_{t-2}$ that caps $H_{t-2} / H_{t-1}$. Hence, $H_{t-2}=H_{t-1} \omega_{\mathrm{e}}(x)=\Psi_{\mathrm{e}}\left(H_{t-2}\right)$. We assume that inductively $\Psi_{\mathrm{e}}\left(H_{1}\right)$ is the product of the caps of all nd-Frattini chief factors in the $G$-chief series $\{1\}=H_{t} \subset \cdots \subset H_{1} \subset N$. If $N / H_{1}$ is d-Frattini, then $\Psi_{\mathrm{e}}\left(H_{1}\right)=\Psi_{\mathrm{e}}(N)$. If $N / H_{1}$ is nd-Frattini, set

$$
U=\Psi_{\mathrm{e}}\left(H_{1}\right) \prod\left\{\omega_{\mathrm{e}}(x) \mid \omega_{\mathrm{e}}(x) \text { caps } N / H_{1}\right\} \unlhd N .
$$

If $U \neq \Psi_{\mathrm{e}}(N)$, there exists a cyclic subgroup $\left\langle x \mid x^{n}=1\right\rangle \subseteq N \backslash U$ for $n \in \mathbb{Q}$. Since $\omega_{\mathrm{e}}(x) \nsubseteq H_{1}$, we find that $\omega_{\mathrm{e}}(x)$ caps $N / H_{1}$. Therefore, $U=\Psi_{\mathrm{e}}(N)$.

Proposition 2.9. For each $G$-chief series contained in a $G$-normal subgroup $N, \Psi_{\mathrm{e}}(N)$ is the product of the caps to the nd-Frattini chief factors in that series. 


\section{3. $\delta$-sets}

This section is the motivation for this study.

Let $\mathcal{X}_{p}$ denote a functor on the $G$-chief factors of a group $G$ such that $\mathcal{X}_{p}(G)$ is the collection of all nd-Frattini chief factors of the form $\omega_{\mathrm{e}}(x) / T=F$ and of order divisible by the prime $p$. Then $\mathcal{X}_{p}(G)=\emptyset$ only if $p \nmid|G|$, which includes all groups of order one. Set

$$
\eta_{p}(G)=\bigcap_{F \in \mathcal{X}_{p}} \mathcal{C}_{G}(F)
$$

and set $\Psi_{p}(G)$ equal to $\prod\left\{\omega_{\mathrm{e}}(x)\right\}$, the product over all subgroups $\left\langle x \mid x^{n}=1\right\rangle \subseteq G$ such that $n=p$ if $p$ is odd or $n \mid 4$ if $p=2$. Then $\Psi_{\mathrm{e}}(G)=\prod_{p \in \pi(G)} \Psi_{p}(G)$, where $\pi(G)$ is the collection of primes dividing $|G|$.

In [2, Theorem A.13.8 (a)], the centralizer $C_{p}$ of all $G$-chief factors with orders divisible by a prime $p$ in a group $G$ and the centralizer $C_{p}^{*}$ of all such factors which are not Frattini are proven to satisfy $C_{p}=C_{p}^{*}=O_{p^{\prime}, p}(G)$, the maximal $G$-normal $p$-nilpotent subgroup in $G$. Laue [6, Lemma 1] has provided a 'dual' $D_{p}$ to $C_{p}, D_{p}$ being the centralizer of all $G$-chief factors in $\Psi_{p}(G)$ of order divisible by the prime $p$, and proved $D_{p}=O_{p^{\prime}, p}(G)$. This result will be extended to the centralizer $D_{p}^{*}$ of the subset that consists only of the nd-Frattini chief factors so that $D_{p}=D_{p}^{*}=\eta_{p}(G)=O_{p^{\prime}, p}(G)$.

By the definition of $\Psi_{p}(G)$, there exists at least one nd-Frattini chief factor with order divisible by $p$. For each such $G$-chief factor $H / K, H=K \omega_{\mathrm{e}}(x)$ for $|x|=p$ or $|x|$ divides 4 . Hence, $H / K \cong_{G} \omega_{\mathrm{e}}(x) / K \cap \omega_{\mathrm{e}}(x)$ implies that $\mathcal{C}_{G}(H / K)=\mathcal{C}_{G}\left(\omega_{\mathrm{e}}(x) / K \cap \omega_{\mathrm{e}}(x)\right)$. Therefore, $\eta_{p}(G) \subseteq D_{p}^{*}$. On the other hand, since each $G$-chief factor $\omega_{\mathrm{e}}(x) / K$ is an nd-Frattini chief factor in $\Psi_{p}(G), D_{p}^{*} \subseteq \eta_{p}(G)$. The following proposition gives a summary.

Proposition 3.1. $D_{p}^{*}=\eta_{p}(G)$ for each group $G$.

The proof of the next result is an analogue of that for [2, Theorem A.13.8 (a)].

Proposition 3.2. $D_{p}^{*}=O_{p^{\prime}, p}(G)$ for each group $G$.

Proof. Suppose that $O_{p^{\prime}, p}(G)=\{1\}$. No minimal $G$-normal subgroup can be abelian. For each such subgroup $N, N$ is an nd-Frattini chief factor, $|N|$ is divisible by the prime $p$, and $\mathcal{C}_{G}(N) \cap N=\{1\}$. Since $D_{p}^{*} \subseteq \mathcal{C}_{G}(N)$ for each $N$, we have $D_{p}^{*} \cap N=\{1\}$, even if $N \subseteq D_{p}^{*}$. From the contradiction we obtain $D_{p}^{*}=\{1\}=O_{p^{\prime}, p}(G)$.

Consider $\{1\} \neq O_{p^{\prime}, p}(G) \neq G$. There exists a minimal $G$-normal subgroup $N \subseteq$ $O_{p^{\prime}, p}(G)=D_{p} \subseteq D_{p}^{*}$. By Proposition 2.4, $D_{p}^{*} / N$ is contained in the centralizer $D / N$ of the nd-Frattini chief factors in $G / N$ having order divisible by $p$. Then $D / N$ is $p$ nilpotent by induction on the group order. So $D_{p}^{*} / N$ is $p$-nilpotent. If $N$ is a $p^{\prime}$-subgroup, then $D_{p}^{*}$ is $p$-nilpotent. If $N$ is a $p$-subgroup, then $N$, as an nd-Frattini chief factor with order divisible by $p$, is contained in $Z\left(D_{p}^{*}\right)$. So $D_{p}^{*}$ is a $p$-soluble group in which all $D_{p}^{*}$ chief factors are central in $D_{p}^{*}$. By $\left[2\right.$, Proposition A.13.3], $D_{p}^{*}$ is $p$-nilpotent. Therefore, $D_{p}^{*} \subseteq O_{p^{\prime}, p}(G)$ implies equality.

Since $O_{p^{\prime}, p}(G)=D_{p}$ by $[\mathbf{6}$, Lemma 1$], D_{p} \subseteq D_{p}^{*}$ implies $O_{p^{\prime}, p}(G)=D_{p}^{*}$ whenever $G=$ $O_{p^{\prime}, p}(G)$. This completes the proof. 
With respect to the above notation, the following corollary is valid.

Corollary 3.3. For a group $G, O_{p^{\prime}, p}(G)=C_{p}=C_{p}^{*}=D_{p}=D_{p}^{*}=\eta_{p}(G)$.

Proof. The first two equalities are valid by [2, Theorem A.13.8 (a)], the third follows from [6, Lemma 1], the fourth by Proposition 3.2, and the last by Proposition 3.1.

A local function $f: \mathbb{P} \rightarrow$ homomorphs is a formation function if $f(p)$ is a formation for each $p \in \mathbb{P}$ with support $\operatorname{Supp}(f) \subseteq \mathbb{P}$ for which $\operatorname{Supp}(f)=\{p \in \mathbb{P} \mid f(p) \neq \emptyset\}$. A $G$-chief factor $H / K$ in a group $G$ is $f$-central if $\operatorname{Aut}_{G}(H / K) \in f(p)$ for each prime $p$ dividing $|H / K|$ and $f$-eccentric otherwise. The local formation $\operatorname{LF}(f)$ is the collection of all groups for which $G \in \operatorname{LF}(f)$ if and only if each non-Frattini $G$-chief factor is $f$-central. As is known, this extends to $G \in \operatorname{LF}(f)$ if and only if each $G$-chief factor is $f$-central (see [2, Theorem IV.3.2.]). It is known that $\operatorname{LF}(f)$ is a saturated formation [2, Theorem IV.3.3], i.e. $G \in \operatorname{LF}(f)$ if and only if $G / \Phi(G) \in \operatorname{LF}(f)$.

Denote by $\delta(f)$ the collection of groups such that $G \in \delta(f)$ if and only if each nd-Frattini chief factor $H / K$ is $f$-central in $G$. We refer to $\delta(f)$ as a $\delta$-set.

Corollary 3.4. With respect to the local function $f$ and within $\operatorname{Supp}(f), \mathfrak{F}=\operatorname{LF}(f)$ is a local formation if and only if $\mathfrak{F}=\delta(f)$. Moreover, a group $G \in \mathfrak{F}$ if and only if $\Psi_{\mathrm{e}}(G) \subseteq Z_{\mathfrak{F}}(G)$, the $\mathfrak{F}$-hypercentre of $G$.

Proof. The first conclusion is a direct application of Corollary 3.3. Since each ndFrattini chief factor $H / K$ is $G$-isomorphic to $\omega_{\mathrm{e}}(x) / K \cap \omega_{\mathrm{e}}(x)$ with respect to a cap $\omega_{\mathrm{e}}(x)$ to $K$ in $H$,

$$
\operatorname{Aut}_{G}(H / K)=\operatorname{Aut}_{G}\left(\frac{\omega_{\mathrm{e}}(x)}{K \cap \omega_{\mathrm{e}}(x)}\right) .
$$

Remark 3.5. Corollary 3.4 extends the list of equivalent conditions stated in $[\mathbf{2}$, Theorem IV.3.2.] with regard to local formations, as follows.

Let $f$ be a formation function and let $\pi$ denote the support of $f$. Any two of the following statements are equivalent:

(a) $G \in \operatorname{LF}(f)$;

(b) $G \in \mathfrak{G}_{\pi} \cap \mathfrak{G}_{\pi^{\prime}} \mathfrak{S}_{p} f(p)$ for all $p \in \pi$;

(c) all chief factors of $G$ are $f$-central.

\section{References}

1. C. Christensen, Groups with complemented normal subgroups, J. Lond. Math. Soc. 42 (1967), 208-216.

2. K. Doerk And T. Hawkes, Finite soluble groups (Walter de Gruyter, New York, 1992).

3. T. HAwKEs, Two applications of twisted wreath products to finite soluble groups, Trans. Am. Math. Soc. 214 (1975), 325-335. 
4. B. Huppert, Enliche Gruppen, I (Springer, 1967).

5. C. JuAn Martínez, Groups with complemented normal subgroups, Doctoral Thesis, Universidad de Valencia (1994).

6. R. Laue, Dualization of saturation for locally defined formations, J. Alg. 52 (1978), 347-353. 